Rapid Fecal Calprotectin Test and Symptom Index in Monitoring the Disease Activity in Colonic Inflammatory Bowel Disease

\title{
Puolanne, Anna-Maija
}

2017-11

Puolanne , A-M , Kolho , K-L , Alfthan , H , Ristimäki , A , Mustonen , H \& Färkkilä , M 2017 , ' Rapid Fecal Calprotectin Test and Symptom Index in Monitoring the Disease Activity in Colonic Inflammatory Bowel Disease ' , Digestive Diseases and Sciences , vol. 62 , no. 11 , pp. 3123-3130 . https://doi.org/10.1007/s10620-017-4770-0

http://hdl.handle.net/10138/260143

https://doi.org/10.1007/s10620-017-4770-0

publishedVersion

Downloaded from Helda, University of Helsinki institutional repository.

This is an electronic reprint of the original article.

This reprint may differ from the original in pagination and typographic detail.

Please cite the original version. 


\title{
Rapid Fecal Calprotectin Test and Symptom Index in Monitoring the Disease Activity in Colonic Inflammatory Bowel Disease
}

\author{
Anna-Maija Puolanne ${ }^{1}$ (1) Kaija-Leena Kolho $^{2} \cdot$ Henrik Alfthan $^{3} \cdot$ \\ Ari Ristimäki ${ }^{4} \cdot$ Harri Mustonen ${ }^{5}$ Martti Färkkilä ${ }^{1}$
}

Received: 25 April 2017/Accepted: 16 September 2017/Published online: 25 September 2017

(C) Springer Science+Business Media, LLC 2017

\begin{abstract}
Background Fecal calprotectin is a reliable surrogate marker for inflammatory activity in inflammatory bowel disease (IBD).

Aims For the noninvasive monitoring of the activity of colonic inflammation, we validated a symptom index suitable for ulcerative colitis and colonic Crohn's disease. By combining the symptom index with a rapid semiquantitative calprotectin test, we constructed a new activity index based on the highest AUCs, using histological remission as a reference. We also evaluated the correlation of the patient-reported influence of the IBD in the daily life, measured by a VAS, with the inflammation activity. Methods The disease activity of 72 patients with IBD of the colon was determined by endoscopic activity scores (SES-CD/UCEIS). The patients provided stool samples for determination of calprotectin and filled in a questionnaire about their symptoms during the last week.
\end{abstract}

\section{Anna-Maija Puolanne}

anna-maija.puolanne@hus.fi;

anna-maija.puolanne@helsinki.fi

Kaija-Leena Kolho

kaija-leena.kolho@hus.fi

Henrik Alfthan

henrik.alfthan@hus.fi

Ari Ristimäki

ari.ristimaki@hus.fi

Harri Mustonen

harri.mustonen@helsinki.fi

Martti Färkkilä

martti.farkkila@hus.fi

1 Department of Medicine, Clinic of Gastroenterology,

Helsinki University Hospital, Haartmaninkatu 4,

P.O. Box 340, 00290 Helsinki, Finland
Results The results of the symptom index demonstrated a statistically significant correlation with the rapid calprotectin test, histological inflammation activity, and the VAS. No correlations were found between the VAS and calprotectin or the histological inflammation activity. The sensitivity of the combination index to detect active inflammation was slightly superior to fecal calprotectin alone.

Conclusion The new symptom index and the combination index are simple, noninvasive means for distinguishing remission from active inflammation in colonic IBD. With the VAS, we can pick up patients who need psychosocial support because of the disease burden, even if their IBD is in remission.

Keywords Inflammatory bowel disease $\cdot$ Fecal calprotectin $\cdot$ Symptom index $\cdot$ Rapid fecal calprotectin test

2 Children's Hospital, Helsinki University Hospital, Stenbäckinkatu 11, 00290 Helsinki, Finland

3 Department of Clinical Chemistry, Helsinki University Hospital, Haartmaninkatu 2, 00290 Helsinki, Finland

4 Pathology, Research Programs Unit and HUSLAB, University of Helsinki and Helsinki University Hospital, Haartmaninkatu 3, 00290 Helsinki, Finland

5 Department of Surgery, Biomedicum Helsinki, University of Helsinki and Helsinki University Hospital, Haartmaninkatu 8, 00290 Helsinki, Finland 


\section{Introduction}

Inflammatory bowel diseases (IBD), ulcerative colitis (UC), and Crohn's disease (CD) are chronic, idiopathic gastrointestinal diseases with alternating relapses and remissions. The goals of therapy in IBD are the control of patients' symptoms and mucosal healing. The evaluation of the inflammatory activity and prediction of relapses are essential for individual therapy and for empowerment patients for disease care. Moreover, continuous inflammation negatively affects the patient's quality of life [1].

Several clinical activity and symptom scores have been developed to help the physicians assess the clinical activity of IBD. Most of the activity scores used in clinical trials are either only symptom-based or too complex and time-consuming for clinical use [2, 3]. In everyday practice, the disease activity is usually determined according to the patient's symptoms, laboratory parameters, or simpler clinical activity indices, such as Simple Clinical Colitis Activity Index (SCCAI) [4] or partial Mayo score without endoscopic subscore for UC [5] and Harvey-Bradshaw index (HBI) for CD [6]. Most scores include a physician's assessment of the disease activity or the physical examination, and they cannot be completed by patients themselves [7].

Fecal calprotectin (FC) is the most widely used surrogate marker for monitoring inflammatory activity. Calprotectin is the main cytosolic protein of the neutrophils and is secreted to the feces by the neutrophils migrating to the bowel lumen through the inflamed bowel wall. The concentration of calprotectin in stools correlates well with ${ }^{111}$ indium-labeled leukocytes [8], and FC has shown to be an excellent marker of mucosal healing [9]. Elevated FC predicts relapse, especially in the next 12 months, while normal FC predicts sustained remission [10, 11]. To make the fecal testing more feasible, several rapid FC tests have been recently developed [12-18].

Irritable bowel syndrome (IBS)-like symptoms in IBD patients are common. In different studies, the prevalence has varied between 9 and $46 \%$ in UC patients, and 42 and $57 \%$ in CD patients with clinical remission, which is two to three times higher than in the normal population [19-21]. Even with the clinical activity scores, distinguishing IBS symptoms from active IBD can be difficult. IBD patients with IBS have reported a lower health-related quality of life, and more anxiety and depression than IBD patients without IBS [19, 22]. In the study of Haapamäki, more than a half of the IBD patients reported that their physicians never ask about the impact of their symptoms on their daily life [22].

Over the past years, clinical research has moved from disease-related outcomes to patient-reported outcomes
(PROs) as primary endpoints. A PRO is a report about a health condition coming directly from a patient without interpretation by anyone else [23]. A typical PRO is a symptom questionnaire filled in by a patient.

In our previous study, we have validated a new rapid semi-quantitative FC test (Prevent ID CalDetect, Preventis, Immunodiagnostics AG, Bensheim, Germany) for detecting the inflammatory activity of colonic IBD, by comparing it with clinical and endoscopic indices, histology, and FC determined with ELISA (enzyme-linked immunosorbent assay) [24]. We demonstrated that CalDetect results correlated significantly with ELISA FC, and they had a close correlation with clinical and endoscopic indices, especially in UC. The diagnostic accuracy of CalDetect for distinguishing remission or mild histological inflammation from active inflammation was comparable with that of ELISA FC.

The aim of the present study was to construct an index, which predicts effectively histological remission in IBD. We validated a new simple symptom score suitable for both UC and colonic CD, and develop a global activity score for colonic IBD by combining the symptom index with a rapid FC test. The secondary goal was to correlate the patient-reported symptoms of the IBD on the daily life and inflammatory activity.

\section{Subjects and Methods}

Patients with colonic IBD from the Helsinki University Hospital and Children's Hospital, University of Helsinki, Helsinki, Finland, referred for an ileocolonoscopy from January 2013 to September 2013, were invited to participate in the study. Exclusion criteria included the presence of isolated disease in the rectum, upper gastrointestinal tract, or ileum; colectomy or extensive colon resection; use of antibiotics at the time of the colonoscopy; or the inability to provide a fecal sample within 30 days of the colonoscopy. The study population consisted of the same patients as in our previous study concerning the rapid fecal tests [24].

The clinical activity of the disease was scored by the endoscopist performing the colonoscopy, according to the partial Mayo score [5] for UC and the Harvey-Bradshaw index (HBI) [25] for CD, and the endoscopic activity according to the UCEIS (Ulcerative Colitis Endoscopic Index for Severity) [26] for UC and the SES-CD (Simple Endoscopic Score for Crohn's Disease) [27] for CD. Two gastroenterologists (M.F. and A.-M.P.) retrospectively evaluated the findings.

The histological inflammation activity was assessed according to the normal clinical routine by experienced pathologists with a scale from zero to three, in which 
$0=$ histological remission, $1=$ mild activity (lamina propria or intraepithelial neutrophils), $2=$ moderate activity (presence of crypt abscess), and $3=$ strong activity (presence of erosion or ulcer) [28].

All patients provided a single fecal sample within 30 days of the colonoscopy. The samples were analyzed in the routine clinical laboratory with an ELISA test (PhiCal Test, Calpro AS, Oslo, Norway) and with a semi-quantitative rapid test for FC (Prevent ID CalDetect, Preventis, Immunodiagnostics AG, Bensheim, Germany; named CalDetect below), according to the manufacturer's instructions. The cutoffs of CalDetect were the $\mathrm{FC}<50 \mu \mathrm{g} / \mathrm{g}, 50-200 \mu \mathrm{g} / \mathrm{g}$ or $>200 \mu \mathrm{g} / \mathrm{g}$.

At the time of colonoscopy, the patients filled in a questionnaire about their symptoms (IBD symptom index, IBD-SI) during the last 7 days. The clinical activity of the disease was determined with 6 questions: general wellbeing, abdominal pain, daily bowel movements, nocturnal bowel movements, presence of blood in stools, and the influence of the IBD on the daily life, which was assessed by visual analog scale (VAS) (Table 2).

To make the noninvasive monitoring of the activity of the colonic IBD even more reliable than with FC or clinical indices alone, we developed a simple combination index using the partial symptom index and CalDetect. The sensitivity and specificity of this new IBD index in differentiating remission and active disease were then compared to the clinical and endoscopic indices and FC results. We wanted the combination index to be simple in the clinical practice, suitable for assessing both $\mathrm{UC}$ and colonic $\mathrm{CD}$, and suitable also for predicting the risk of relapse. The relapse risk correlates better with the histological than clinical or endoscopic inflammation activity. For that reason, we chose the histological inflammation activity as the endpoint of the new score, instead of the clinical or endoscopic indices.

\section{Statistics}

Results are given as the number of patients (percentage), and continuous results are given as median (range). The Spearman's rank correlation coefficient rho $(\rho)$ was calculated to assess correlations between the two variables. The receiver operating characteristic (ROC) curves were drawn, and area under curve (AUC) was calculated. The logistic multivariate regression was used to find out initial coefficients for the simple formula to detect disease activity. Results were analyzed with IBM SPSS (version 24, IBM Corp, New York) and R-software (www.r-project. org).

\section{Ethical Considerations}

All patients provided their written informed consent prior to enrollment, and the Ethics Committee of the Helsinki University Hospital, Helsinki, Finland, approved the study protocol.

\section{Results}

\section{Patients}

Of the evaluated 132 patients, 72 fulfilled the inclusion criteria. Of the included patients, 50 had UC and 22 had CD. Of the 60 excluded patients, 8 had undergone extensive colon resection or colectomy; 17 presented with isolated ileitis or proctitis, or gastrointestinal disease other than IBD; 13 provided no stool sample; and 22 failed to fulfill the inclusion criteria for other reasons (e.g., incomplete colonoscopy, no biopsies taken or use of antibiotics at the time of the colonoscopy). The clinical and demographic data, FC values, and the IBD activity indices of the included patients are given in Table 1 .

\section{Symptoms}

For the analysis, we divided the total symptom index into two parts: partial IBD symptom index without the VAS (pIBD-SI) including the patient-reported assessment of the IBD activity (Questions 1-5, Table 2) and the VAS (IBDVAS) measuring the influence of IBD on the patient's daily life (Question 6, Table 2). The most reported symptoms were general well-being below normal and abdominal pain, and the less reported symptom was nocturnal bowel movements.

The results of the analysis of the symptom index of the included patients are given in Table 3 .

The median pIBD-SI in all patients was 0 points (0-9) and demonstrated weak but statistically significant correlation with both CalDetect $(\rho=0.249, p=0.05)$ and ELISA FC $(\rho=0.235, p<0.05)$. The correlations of the pIBD-SI with the histological inflammation activity and the IBD-VAS were also statistically significant ( $\rho=0.308$ and 0.677 , respectively, $p<0.05$ ), as well as the correlation between pIBD-SI and UCEIS $(\rho=0.506, p<0.001)$. In CD patients, pIBD-SI did not correlate with the endoscopic activity. The highest sensitivity of the partial symptom index for identifying remission or mild inflammation was $73 \%$ and the specificity $72 \%$.

The median IBD-VAS of all patients was 3 points (1-7). We found no significant correlations between the VAS and CalDetect or the histological inflammation activity. The 
Table 1 Clinical and demographic data, FC values, and the IBD activity indices of the included patients

\begin{tabular}{|c|c|c|c|}
\hline & tot, $n=72$ & $\mathrm{CD}, n=22$ & $\mathrm{UC}, n=50$ \\
\hline Gender male, $n(\%)$ & $48(67)$ & $16(72)$ & $32(64)$ \\
\hline Age, years median (range) & $36(3-71)$ & $36(13-60)$ & $36(3-71)$ \\
\hline Disease duration, years median (range) & $8(0-36)$ & $11(0-27)$ & $7(0-36)$ \\
\hline \multicolumn{4}{|l|}{ Medication, $n(\%)$} \\
\hline 5-ASA & $51(71)$ & $13(59)$ & $38(76)$ \\
\hline Thiopurines & $55(76)$ & $12(55)$ & $28(56)$ \\
\hline MTX & $2(3)$ & $0(0)$ & $2(4)$ \\
\hline Steroids & $9(13)$ & $2(9)$ & $7(14)$ \\
\hline IFX/adalimumab & $16(22)$ & $6(27)$ & $10(20)$ \\
\hline CyA/tacro & $3(4)$ & $1(5)$ & $2(4)$ \\
\hline ELISA FC $\mu \mathrm{g} / \mathrm{g}$ median (range) & $65(5-2082)$ & $82(13-1769)$ & $62(5-2082)$ \\
\hline CalDetect $\mu \mathrm{g} / \mathrm{g}$ median & $50-200$ & $>200$ & $50-200$ \\
\hline \multicolumn{4}{|l|}{ Clinical activity, median (range) } \\
\hline HBI & - & $1(0-10)$ & - \\
\hline Partial Mayo & - & - & $4.5(0-9)^{\mathrm{b}}$ \\
\hline \multicolumn{4}{|l|}{ Endoscopic activity, median (range) } \\
\hline SES-CD & - & $0(0-8)$ & - \\
\hline UCEIS & - & - & $3(3-8)^{b}$ \\
\hline Histological inflammation activity, median & 0 & 0 & 1 \\
\hline pIBD-SI, median (range) & $0(0-9)$ & $1(0-7)$ & $1(0-9)^{\mathrm{b}}$ \\
\hline VAS, median (range) & $3(1-7)$ & $2.5(1-7)$ & $3(1-6)$ \\
\hline Physician's assessment, median (range) ${ }^{\mathrm{a}}$ & & $0(0-1)$ & $0(0-3)$ \\
\hline
\end{tabular}

UC: Physician's global assessment (partial Mayo), range 0-3

Only total symptom index in $2 \mathrm{CD}$ patients and $1 \mathrm{UC}$ patient

$F C$ Fecal calprotectin, IBD inflammatory bowel disease, $C D$ Crohn's disease, $U C$ ulcerative colitis; $M T X$ methotrexate, IFX infliximab, CyA cyclosporin, tacro tacrolimus, HBI Harvey-Bradshaw index, SES-CD Simple Endoscopic Score for Crohn's disease, UCEIS Ulcerative Colitis Endoscopic Index for Severity, pIBD-SI partial IBD symptom index, VAS visual analog scale

${ }^{\mathrm{a}} \mathrm{CD}$ : General well-being (HBI), range $0-3$

${ }^{\mathrm{b}}$ A significant correlation $(p<0.001)$ between the partial symptom score and the clinical and endoscopic activity in UC but not CD sensitivity of the IBD-VAS to distinguish histological remission from active inflammation with a cutoff of 2 points was $87 \%$, while the specificity was only $39 \%$. In 1 UC patient and $2 \mathrm{CD}$ patients, the VAS results were not available.

We also evaluated the physician's assessment of the clinical activity of IBD by the partial Mayo score for UC and HBI for CD. The median physician's assessment was 0 points in both $\mathrm{CD}$ and UC patients, meaning clinical remission. It correlated significantly with the patient-reported activity of IBD (pIBD-SI) $(\rho=0.542, p<0.001)$ and with the IBD-VAS $(\rho=0.337, p<0.05)$.

\section{Clinical, Endoscopic, and Histological Inflammation Activity}

The clinical activity was measured by partial Mayo score for UC and HBI for CD. When the cutoffs for remission were partial Mayo score 0 points [5] and $\mathrm{HBI} \leq 4$ points [25], $44 \%$ of UC patients and $91 \%$ of CD patients were in clinical remission.

The majority of patients showed low endoscopic activity, assessed by UCEIS for UC and SES-CD for CD. If the cutoff points for remission are set at 3 points for UCEIS [26] and 2 points for SES-CD [27, 29], 52\% of UC patients and $59 \%$ of CD patients were in endoscopic remission.

Of all included patients, 52\% were in histological remission (histological inflammation activity $=0$ ), and $78 \%$ had histological inflammation activity $0-1$, meaning histological remission or mild inflammation.

\section{Fecal Calprotectin}

The median ELISA FC of included patients was $65 \mu \mathrm{g} / \mathrm{g}$ (range 5-2082), and the median CalDetect value was between 50 and $200 \mu \mathrm{g} / \mathrm{g}$. Measured by ELISA FC, $60 \%$ of 
Table 2 IBD symptom index (IBD-SI) questionnaire

\begin{tabular}{|c|c|}
\hline \multirow[t]{4}{*}{ 1. General well-being } & 0. Very well \\
\hline & 1. Slightly below normal \\
\hline & 2. Poor \\
\hline & 3. Very poor \\
\hline \multirow[t]{4}{*}{ 2. Abdominal pain } & 0. None \\
\hline & 1. Mild pain \\
\hline & 2. Moderate pain \\
\hline & 3. Severe pain \\
\hline \multirow[t]{4}{*}{ 3. Daily bowel movements } & 0. Normal \\
\hline & 1. 1-2 times more than normal \\
\hline & 2. 3-4 times more than normal \\
\hline & 3. 5 or more times more than norma \\
\hline \multirow[t]{4}{*}{ 4. Nocturnal bowel movements } & 0. normal \\
\hline & 1. 1-2 times more than normal \\
\hline & 2. 3-4 times more than normal \\
\hline & 3. 5 or more times more than normal \\
\hline \multirow[t]{4}{*}{ 5. Presence of blood in stools } & 0. None \\
\hline & 1. Strikes of blood \\
\hline & 2. Obvious blood \\
\hline & 3. Blood alone \\
\hline 6. Influence of the symptoms or & VAS from 1 (very little) to 7 (very $n$ \\
\hline
\end{tabular}

$V A S$ Visual analog scale
Table 3 Results of the partial symptom index and IBD-VAS in comparison of the FC values

\begin{tabular}{|c|c|c|c|c|c|c|}
\hline & \multicolumn{3}{|c|}{ pIBD-SI, median (range) } & \multicolumn{3}{|c|}{ IBD-VAS, median (range) } \\
\hline & Tot & $\mathrm{CD}$ & UC & Tot & $\mathrm{CD}$ & UC \\
\hline \multicolumn{7}{|c|}{ ELISA FC, $\mu \mathrm{g} / \mathrm{g}$} \\
\hline$<50$ & $0.5(0-8)$ & $2(0-7)$ & $0(0-3)$ & $2(1-6)$ & $4(1-7)$ & $1(1-5)$ \\
\hline 50-200 & $0(0-7)$ & $1(0-6)$ & $1(0-3)$ & $4(1-7)$ & $2(1-5)$ & $3(1-5)$ \\
\hline$>200$ & $2(0-9)$ & $0(0-2)$ & $2(0-9)$ & $3(1-6)$ & $3(1-5)$ & $4(1-6)$ \\
\hline \multicolumn{7}{|c|}{ CalDetect, $\mu \mathrm{g} / \mathrm{g}$} \\
\hline$<50$ & $1(0-8)$ & $2(0-2)$ & $0(0-2)$ & $2(1-6)$ & $5(1-6)$ & $1(1-5)$ \\
\hline $50-200$ & $0(0-7)$ & $1(0-7)$ & $0(0-3)$ & $3(1-7)$ & $2(1-7)$ & $3(1-5)$ \\
\hline$>200$ & $1(0-9)$ & $0(0-2)$ & $0(0-9)$ & $3(1-6)$ & $3(1-5)$ & $4(1-6)$ \\
\hline
\end{tabular}

Total number of patients $=69, \mathrm{CD}$ patients $n=20$, UC patients $n=49$

pIBD-SI Partial IBD symptom index, $F C$ fecal calprotectin, $C D$ Crohn's disease, $U C$ ulcerative colitis

$p<0.05$ for the correlation between the pIBD-SI and ELISA FC, CalDetect, and IBD-VAS

all patients were in remission (the routine $\mathrm{FC}$ cutoff value of $100 \mu \mathrm{g} / \mathrm{g}$ for remission [30-32]).

\section{Combination Index}

A formula with a combination of the rapid semi-quantitative fecal calprotectin test and symptom score was constructed giving the highest AUCs (area under the curve) using histological remission or mild inflammation (histological activity $0-1$ ) as a reference. To construct a simple formula, the initial coefficients were obtained from logistic regression, and the combination with the highest AUC was selected. The highest AUC was achieved using the following formula:

$$
\begin{aligned}
& 2.0(\text { if pIBD-SI is } 1 \text { or } 2 \text { points })+3.4(\text { if } \text { pIBD-SI }>2 \text { points }) \\
& +1.0(\text { if CalDetect is } 50-200 \mu \mathrm{g} / \mathrm{g}) \\
& +2.5(\text { if Caldetect }>200 \mu \mathrm{g} / \mathrm{g})
\end{aligned}
$$

The median pIBD-SI-C of all patients was 2.5 points (0-5.9 points). The AUC of the pIBD-SI-C for detecting histological remission or mild disease (histological activity $0-1)$ was 0.822 , compared with the AUC of 0.755 of CalDetect alone and the AUC of 0.628 of the pIBD-SI alone (Fig. 1). If we set the cutoff point at 2.5 points, the sensitivity of the pIBD-SI-C to differentiate remission or mild disease from active inflammation was $93 \%$ and the specificity was $61 \%$.

\section{Discussion}

In this study, we validated a new simple symptom index for both UC and colonic CD designed for everyday practice and to be completed by the patient, by comparing it with 
clinical indices (partial Mayo score for UC and HBI for $\mathrm{CD}$ ), endoscopic indices (UCEIS for UC and SES-CD for $\mathrm{CD})$, as well as FC measured by ELISA and a semiquantitative rapid FC test CalDetect. The index was divided into two parts: partial IBD symptom index without the VAS (pIBD-SI) and the VAS. We also developed a new combination index (pIBD-SI-C) by combining the partial symptom score and CalDetect for evaluation of inflammatory activity of both UC and colonic $\mathrm{CD}$, and we compared the sensitivity and specificity of this combination index in evaluating IBD activity to histological inflammation activity. We also evaluated the influence of IBD on the patient's everyday life (IBD-VAS) correlated with the clinical, endoscopic, and histological activity and FC, and how the patient's and physician's assessments of the influence of IBD on the patient's daily life correlated with each other.

We found that the results of the patient-reported assessment of the IBD activity measured with pIBD-SI correlated significantly with histological inflammation activity and with FC measured by CalDetect and ELISA. The sensitivity of pIBD-SI for detecting remission or mild disease at a cutoff of 2 points was comparable to those of CalDetect and ELISA FC at a cutoff of $200 \mu \mathrm{g} / \mathrm{g}$, and it was slightly superior to that of ELISA FC at a routine cutoff of $100 \mu \mathrm{g} / \mathrm{g}$ (Table 4). When UC and CD patients were analyzed separately, the correlations with clinical and endoscopic activity and FC were stronger in UC patients.
The sensitivity of the pIBD-SI-C with the cutoff of 2.5 points in detecting remission was somewhat higher than those of CalDetect and ELISA FC alone. In this study, 28\% of all patients had CalDetect less than $50 \mu \mathrm{g} / \mathrm{g}$, but this cutoff has proved to be too low for clinical practice in IBD patients [33]. This has also been shown in our previous study, in which the diagnostic accuracy for remission of the CalDetect result $<50 \mu \mathrm{g} / \mathrm{g}$ was only $46 \%$ with the specificity of $34 \%$ [24].

If the physician's assessment of the patient's well-being is based only on the clinical or laboratory parameters, it may differ markedly from the patient's view [34]. In the present study, the influence of the IBD on the patient's everyday life (IBD-VAS) correlated surprisingly well with the physician's view of the IBD activity, the symptom index, and with the clinical and endoscopic indices in UC patients. However, the correlation between the IBD-VAS and FC results, and in CD patients' clinical or endoscopic indices, was not significant. The poor correlation between the endoscopic activity and health-related quality of life in CD patients, regardless of the disease location or behavior, has also been seen in a recent study [1]. A significant proportion of $\mathrm{CD}$ patients also have other than colonic involvement of the disease [35]. In CD, there is a deeper inflammation of the bowel wall, and this systemic inflammation may affect the patient's quality of life more than mucosal inflammation in UC. According to some studies, CD patients have more IBS symptoms than UC patients
Fig. 1 ROC curves for CalDetect, ELISA FC, partial IBD symptom index (pIBD-SI), combination index (pIBD-SIC), IBD-VAS, and the physician's assessment in detecting histologically active inflammation

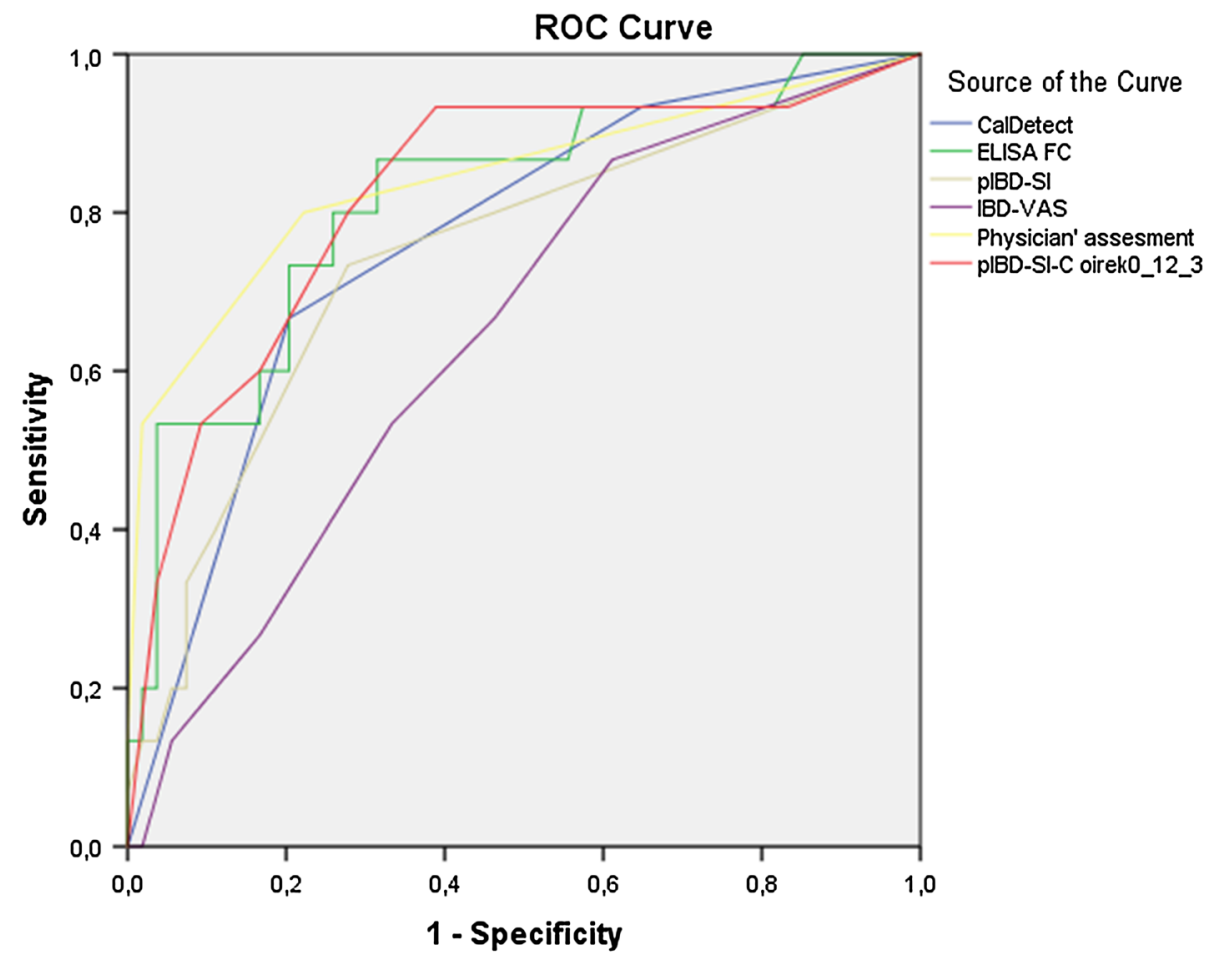

Diagonal segments are produced by ties. 
Table 4 Comparison of the sensitivities and specificities of symptom indices and fecal calprotectin to detect active inflammation

\begin{tabular}{llll}
\hline Variable & Cutoff & Sensitivity, $\%$ & Specificity, \% \\
\hline pIBD-SI & 2 points & 73 & 72 \\
pIBD-SI-C & 2 points & 93 & 61 \\
VAS & 2 points & 87 & 39 \\
ELISA FC & $100 \mu \mathrm{g} / \mathrm{g}$ & 81 & 68 \\
& $200 \mu \mathrm{g} / \mathrm{g}$ & 69 & 75 \\
CalDetect & $200 \mu \mathrm{g} / \mathrm{g}$ & 67 & 79 \\
\hline
\end{tabular}

pIBD-SI Partial IBD symptom index, FC fecal calprotectin, $p I B D-S I$ $C$ partial IBD symptom index with CalDetect

[20], and the health-related quality of life in general is lower in CD than in UC patients [28]. This may explain the difference between $\mathrm{CD}$ and UC groups. We did not ask the patients to specify how IBD affects their life. The VAS cannot be validated and it is not specific for gastrointestinal symptoms, or even organic symptoms, but can even reflect a patient's fear of disease flare or cancer or any psychosocial problems caused by IBD.

In recent studies, new combination scores were developed by combining the results of HBI in CD patients, and SCCAI in UC patients, and FC measured by ELISA or a rapid home test [3, 36, 37]. Our new combination index is feasible for both UC and CD patients with colonic disease, and it correlated significantly with the clinical activity of colitis. The AUC of the combination index for detecting histological remission or mild disease was superior to CalDetect or ELISA FC alone, but not significantly.

We are aware of the limitations of our study. Because the FC concentration depends on the location of the inflammation and is higher especially in rectal but also in colonic than in ileal inflammation $[2,35]$, we included only patients with colonic IBD. We recruited consecutive UC and $\mathrm{CD}$ patients referred to the colonoscopy for any reason regardless of the disease activity, and thus, a significant number of the patients were in remission. For the same reason, the study population comprised only $22 \mathrm{CD}$ patients, which may have skewed the results. Because the demographic data were collected retrospectively from the medical records and not directly from the patients, we did not know whether the patients were using medication affecting the FC result (e.g., non-steroidal anti-inflammatory drugs). Collection of the stool samples was not performed exactly at the time of the colonoscopy, mostly due to the compliance matters, but no major changes in the medication were done between the colonoscopy and the stool sample collection.

The treatment and monitoring of IBD have changed during past decades, and it is moving toward more personalized and individually tailored care, giving more responsibility to the patient. Traditionally, the evaluation of the inflammation activity of IBD has been based on the endoscopy, which is invasive, time-consuming, and unpleasant, and contains a small risk of complications. After the introduction of commercially available FC tests, the role of the endoscopy in detecting the relapse and monitoring the treatment response has become less important. If the inflammation activity could be reliably and objectively monitored noninvasively, it could spare the endoscopy resources. The new pIBD-SI is a rapid way to assess the clinical activity of both UC and colonic CD. It is reliable, and its sensitivity and specificity to distinguish remission from active inflammation are comparable to the rapid FC test CalDetect. In contrast to other IBD activity scores, the patient can complete pIBD-SI him/herself. By combining the FC test with the symptom index, patients can monitor their disease activity at home and contact the outpatient clinic only when needed. In our study, the patient-reported influence of IBD on the daily life (IBDVAS) did not correlate significantly with the FC or histological activity of IBD. However, by this structured evaluation of the influence of the IBD on the patient's life, we can pick up those patients who need psychosocial support because of the disease burden, even if their IBD is in remission. Our study population comprised only patients with colonic IBD. The feasibility of these new symptom indices in the ileal or isolated rectal inflammation needs further investigation.

Acknowledgments We thank our study nurses Pirkko Tuukkala, Virpi Pelkonen, Paula Karlsson, and Anne Nikkonen for their assistance. A.-M.P. designed the study, participated in the data analysis, and drafted the manuscript. K.-L. K. participated in designing the study, data analysis, and manuscript drafting. H.A. performed and analyzed the rapid fecal tests and participated in manuscript drafting. A.R. performed the histological analysis and scoring of the colonoscopy biopsies and helped to draft the manuscript. H.M performed the data analysis and helped to draft the manuscript. M.F. designed and supervised the study and participated in the data analysis and manuscript drafting. All the authors have read and approved the final manuscript.

Funding This work was supported by Grants from the State funding for university-level health research, the Pediatric Research Foundation, and the Finnish Medical Foundation.

\section{Compliance with ethical standards}

Conflict of interest The authors do not have any conflicts of interest.

\section{References}

1. Taleban S, Stewart KO, Li DK, et al. Clinical activity and quality of life indices are valid across ulcerative colitis but not Crohn's disease phenotypes. Dig Dis Sci. 2016;61:2627-2635. doi:10. 1007/s10620-016-4180-8. 
2. Ricanek P, Brackmann S, Perminow G, et al. Evaluation of disease activity in IBD at the time of diagnosis by the use of clinical, biochemical, and fecal markers. Scand J Gastroenterol. 2011;46:1081-1091.

3. af Björkesten C, Nieminen U, Turunen U, et al. Surrogate markers and clinical indices, alone or combined, as indicators for endoscopic remission in anti-TNF-treated luminal Crohn's disease. Scand J Gastroenterol. 2012;47:528-537.

4. Walmsley RS, Ayres RCS, Pounder RE, et al. A simple clinical colitis activity index. Gut. 1998;43:29-32.

5. Schroeder KW, Tremaine WJ, Ilstup DM. Coated oral 5-aminosalisylic acid therapy for mildly to moderately active ulcerative colitis. A randomized study. $N$ Engl $J$ Med. 1987;317:1625-1629.

6. Vermeire S, Schreiber S, Sandborn WJ, et al. Correlation between the Crohn's disease activity and Harvey-Bradshaw indices in assessing Crohn's disease severity. Clin Gastroenterol Hepatol. 2010;8:357-363.

7. Henao MP, Bewtra M, Osterman MT. Measurement of inflammatory bowel disease symptoms: reliability of an abbreviated approach to data collection. Inflamm Bowel Dis. 2015;21: 2262-2271.

8. Roseth AG, Schmidt PN, Fagerhol MK. Correlation between faecal excretion of indium-111-labeled granulocytes and calprotectin, a granulocyte marker protein, in patients with inflammatory bowel disease. Scand J Gastroenterol. 1999;34:50-54.

9. Molander P, Sipponen T, Kemppainen H, et al. Achievement of deep remission during TNFa-blocking agents in IBD. J Crohn's Colitis. 2013;7:730-735.

10. Tibble JA, Sigthorsson G, Bridger S, et al. Surrogate markers of intestinal inflammation are predictive of relapse in patients with inflammatory bowel disease. Gastroenterology. 2000;119:15-22.

11. Laharie D, Mesli S, El Hajbi F, et al. Prediction of Crohn's disease relapse with faecal calprotectin in infliximab responders: a prospective study. Aliment Pharmacol Ther. 2011;34:462-469.

12. Damms A, Bischoff SC. Validation and clinical significance of a new calprotectin rapid test for the diagnosis of gastrointestinal diseases. Int J Colorectal Dis. 2008;23:985-992.

13. Elkjaer M, Burisch J, Voxen Hansen V, et al. A new rapid home test for faecal calprotectin in ulcerative colitis. Aliment Pharmacol Ther. 2009;31:323-330.

14. Labaere D, Smismans A, Van Olmen A, et al. Comparison of six different calprotectin assays for the assessment of inflammatory bowel disease. UEG J. 2014;2:30-37.

15. Lobatón T, López-García A, Rodríguez-Moranta F, et al. A new rapid test for fecal calprotectin predicts endoscopic remission and postoperative recurrence in Crohn's disease. J Crohn's Colitis. 2013;7:640-651.

16. Lobatón T, Rodríguez-Moranta F, Lopez A, et al. New rapid quantitative test for fecal calprotectin predicts endoscopic activity in ulcerative colitis. Inflamm Bowel Dis. 2013;42:1034-1042.

17. Rogler G, Aldeguer X, Kruis W, et al. Concept for a rapid pointof-care calprotectin diagnostic test for diagnosis and disease activity monitoring in patients with inflammatory bowel disease: expert clinical opinion. J Crohn's Colitis. 2013;7:670-677.

18. Vestergaard TA, Nielsen SL, Dahlerup JF, et al. Fecal calprotectin: assessment of a rapid test. Scand J Clin Lab Invest. 2008;68:343-347.

19. Jonefjäll B, Strid H, Öhman L, et al. Characterization of IBS-like symptoms in patients with ulcerative colitis in clinical remission. Neurogastroenterol Motil. 2013;25:756-e578.

20. Simrén M, Axelsson J, Gillberg R, et al. Quality of life in inflammatory bowel disease in remission: the impact of IBS-like symptoms and associated psychological factors. Am J Gastroenterol. 2002;97:389-396.

21. Keohane J, O’Mahony C, O’Mahony L, et al. Irritable bowel syndrome-type symptoms in patients with inflammatory bowel disease: a real association or reflection of occult inflammation? Am J Gastroenterol. 2010;105:1789-1794.

22. Haapamäki J, Turunen U, Roine RP, et al. Finnish patients with inflammatory bowel disease have fewer symptoms and are more satisfied with their treatment than patients in the previous European survey. Scand J Gastroenterol. 2008;43:821-830.

23. Bodger K, Ormerod C, Shackcloth D, et al. On behalf of the IBD Control Collaborative. Development and validation of a rapid, generic measure of disease control from the patient's perspective: the IBD-Control questionnaire. Gut. 2014;63:1092-1102.

24. Puolanne A, Kolho K, Alfthan H, et al. Rapid faecal tests for detecting disease activity in colonic IBD. Eur J Clin Invest. 2016;46:825-832.

25. Harvey RF, Bradshaw JM. A simple index of Crohn's disease activity. Lancet. 1980;1:514.

26. Travis S, Schnell D, Krzeski P, et al. Developing an instrument to assess the endoscopic severity of ulcerative colitis: the Ulcerative Colitis Endoscopic Index of Severity (UCEIS). Gut. 2011;61:535-542.

27. Schoepfer A, Beglinger C, Straumann A, et al. Fecal calprotectin correlates more closely with the Simple Endoscopic Score for Crohn's disease (SES-CD) than CRP, blood leukocytes, and the CDAI. Am J Gastroenterol. 2010;105:162-169.

28. Magro F, Portela F, Lago P, et al. Inflammatory bowel disease: a patient's and caregiver's perspective. Dig Dis Sci. 2009;54:2671-2679. doi:10.1007/s10620-008-0658-3.

29. Daperno M, D'Haens G, Van Assche G, et al. Development and validation of a new, simplified endoscopic activity score for Crohn's disease: the SES-CD. Gastrointest Endosc. 2004;60:505-512.

30. Sipponen T, Kolho K. Faecal calprotectin in children with clinically quiescent inflammatory bowel disease. Scand J Gastroenterol. 2010;45:872-877.

31. D'Haens G, Sandborn WJ, Feagan BG, et al. A review of activity indices and efficacy end points for clinical trials of medical therapy in adults with ulcerative colitis. Gastroenterology. 2007;132:763-786.

32. Kolho K-L, Raivio T, Lindahl $\mathrm{H}$, et al. Faecal calprotectin remains high during glucocorticoid therapy in children with inflammatory bowel disease. Scand $J$ Gastroenterol. 2006;41:720-725.

33. D'Haens G, Ferrante M, Vermeire S, et al. Fecal calprotectin is a surrogate marker for endoscopic lesions in inflammatory bowel disease. Inflamm Bowel Dis. 2012;18:2218-2224.

34. Mitchell R, Kremer A, Westwood N, et al. Talking about life and IBD: a paradigm for improving patient-physician communication. J Crohn's Colitis. 2009;3:1-3.

35. Sipponen T, Kärkkäinen $\mathrm{P}$, Savilahti E, et al. Correlation of faecal calprotectin and lactoferrin with an endoscopic score for Crohn's disease and histological findings. Aliment Pharmacol Ther. 2008;28:1221-1229.

36. Pedersen N, Elkjaer M, Duricova D, et al. eHealth: individualisation of infliximab treatment and disease course via a selfmanaged web-based solution in Crohn's disease. Aliment Pharmacol Ther. 2012;36:840-849.

37. Elkjaer M, Shuhaibar M, Burisch J, et al. E-health empowers patients with ulcerative colitis: a randomised controlled trial of the web-guided 'Constant-care' approach. Gut. 2010;59: $1652-1661$ 